\title{
Digitized self-oscillating loop for piezoelectric transformer-based power converters
}

\author{
Ekhtiari, Marzieh; Andersen, Thomas; Zhang, Zhe; Andersen, Michael A. E.
}

Published in:

Proceedings of IEEE Applied Power Electronics Conference 2016

Link to article, DOI:

10.1109/APEC.2016.7468056

Publication date:

2016

Document Version

Peer reviewed version

Link back to DTU Orbit

Citation (APA):

Ekhtiari, M., Andersen, T., Zhang, Z., \& Andersen, M. A. E. (2016). Digitized self-oscillating loop for piezoelectric transformer-based power converters. In Proceedings of IEEE Applied Power Electronics Conference 2016 (pp. 1430-1436). IEEE. https://doi.org/10.1109/APEC.2016.7468056

\section{General rights}

Copyright and moral rights for the publications made accessible in the public portal are retained by the authors and/or other copyright owners and it is a condition of accessing publications that users recognise and abide by the legal requirements associated with these rights.

- Users may download and print one copy of any publication from the public portal for the purpose of private study or research.

- You may not further distribute the material or use it for any profit-making activity or commercial gain

- You may freely distribute the URL identifying the publication in the public portal 


\title{
Digitized self-oscillating loop for piezoelectric transformer-based power converters
}

\author{
Marzieh Ekhtiari, Thomas Andersen, Zhe Zhang and Michael A. E. Andersen \\ Electronics Group, Department of Electrical Engineering \\ Technical University of Denmark \\ Richard Petersens Plads, bldg. 325, DK-2800, Kgs. Lyngby, Denmark \\ Web: http://www.ele.elektro.dtu.dk/ \\ Email: maekh@elektro.dtu.dk
}

\begin{abstract}
A new method is implemented in designing of self-oscillating loop for driving piezoelectric transformers. The implemented method is based on combining both analog and digital control systems. Digittzed delay, or digitized phase shift through the self-oscillating loop results in a very precise frequency control and ensures an optimum operation of the piezoelectric transformer in terms of voltage gain and efficiency. In this work, additional time delay is implemented digitally for the first time through $\mathbf{1 6}$ bit digital-to-analog converter to the self-oscillating loop. Delay control setpoints updates at a rate of $417 \mathrm{kHz}$. This allows the control loop to dynamically follow frequency changes of the transformer in each resonant cycle. The operation principle behind self-oscillating is discussed in this paper. Moreover, experimental results are reported.

Index Terms-Optimum delay line; self-oscillating loop; phase shift; switch-mode power supply; zero-voltage switching; plezoelectric transformer.
\end{abstract}

\section{NOMENCLATURE}

BPF Band pass filter.

C Resonant capacitance of the piezoelectric transformer.

$\mathrm{C}_{\mathrm{A}} \quad$ Measurement capacitance.

$C_{d 1} \quad$ Input electrode capacitance of the piezoelectric transformer.

$C_{d 2} \quad$ Output electrode capacitance of the piezoelectric transformer.

CEZC Current estimate zero crossing.

D Duty cycle.

DAC Digital-to-analog converter.

DDL Digitized delay line.

DDL out Output of the DDL.

DT Dead time.

EDDL $_{\text {in }}$ Edge detected of the input of the DDL.

FF Flip-flop.

FPGA Field-programmable gate array. $i_{\text {res }}(\mathrm{t}) \quad$ Piezoelectric transformer's resonant current.

$L \quad$ Internal inductance of the piezoelectric transformer.

LPF Low pass filter.

MOSFET Metal-oxide-semiconductor field-effect transistor.

PT Piezoelectric transformer.

$R \quad$ Dielectric losses inside the transformer.

$\mathrm{R}_{\mathrm{A}}, \mathrm{R}_{\mathrm{B}} \quad$ Sensing resistors.

$R_{L} \quad$ Load resistor.

$R_{\text {marched }} \quad$ Matched load for the piezoelectric transformer

$\mathrm{v}_{\mathrm{FP}}(\mathrm{t}) \quad$ Switching voltage in the primary side of the PT.

ZVS Zero voltage switching.

$\omega \quad$ Angular frequency.

$\phi_{I} \quad$ Phase of the resonant current.

\section{INTRODUCTION}

Piezoelectric transformers' (PT) voltage gain, resonance frequency and efficiency change with variation of their load and temperature [1]-[4]. Therefore, in order to operate a PT in its optimum point, located slightly above the resonant frequency, it is necessary to follow changes in the resonance frequency [1]. It is required that the selfoscillating loop be able to adjust its phase shift to follow the PT's resonant frequency. Therefore, implemented adjustable phase shift compensates for the rest of the phase shift in the loop for frequency variations. For instance, when the energy transfered by the converter needs to be controlled to maintain DC output voltage at different voltage levels, the PT's load changes. Any changes in the PT's load causes a change in its operating point [4]. In order to keep the operation of the PT at its most efficient point, its operating frequency should 
be changed. This is performed by changing the converter's switching frequency. The switching frequency is controlled through a self-oscillating loop. Therefore, by changing the predesigned phase shift, the switching frequency follows variations in the PT's resonant current.

Phase shift compensation with high resolution becomes necessary especially when the load of the converter is variable. These variations directly translates to the PT's load variations. If the total phase shift of the loop is not properly adjusted to an integer factor of $360^{\circ}$, it causes a damping of the resonant current and, the closed-loop operation cannot be achieved, so the converter will not start working. Therefore, very fine resolution for the phase shift adjustment is required.

In previous research, an adjustable time delay block that controls the total phase of the loop has been implemented through an analog circuit. In the closedloop operation, $360^{\circ}$ phase difference in the desired frequency cannot be ensured for the load or temperature variations of the PT [5], [6]. To solve this problem, a digitalized phase shift compensation is applied in this paper. Changes in the PT's resonant frequency are compensated for the closed-loop by detecting and adding required phase shift in order to obtain a full loop phase shift of $360^{\circ}$. Compensation is performed by adding a finely-controlled time delay to the feedback chain. Resolution of the applied time delay is $1 \mathrm{~ns}$. This ensures that the added time delay is finely controllable in order to precisely adapt the frequency of the self-oscillating loop and match changes in the PT's operating point. Furthermore, the delay can work in the range between $0^{\circ}$ to $180^{\circ}$. This then, further ensures soft switching operation of the PT and efficiency increase.

This paper is organized as follows. Section II presents thorough explanation of the self-oscillating loop. Subsections II-A and II-B explain the principle behind selfoscillation in the prior art. Section II-C shows experimental results. Subsection III-A gives more explanation about the proposed method and subsection III-B shows experimental results for the proposed method.

\section{Self-oscillating LOOP FOR PT-BASED CONVERTERS}

\section{A. Principle and design considerations}

Essentially, two requirements need to be satisfied in order to produce sustained oscillation in a closedloop. One is that phase angle of the entire loop should be an integer multiple of $360^{\circ}$; the other requirement is that the loop gain should be greater than unity to start-up the oscillation. The former condition is fulfilled by adjusting phase shift through the loop. The latter condition is fulfilled by the use of a comparator since the comparator's gain can be considered infinite and therefore its input becomes saturated to the rail voltages. This results in generating square waves in the output of the comparator.

Fig. 1 shows the circuit for the self-oscillating loop together with the PT-based power stage with matched load, $R_{\text {marched. }}$. Fig. 2 shows the block diagram for the self-oscillating loop [7]. A small perturbation in the loop starts the self-induced oscillation. This results in selfexcitation of the resonant current inside the PT during the start-up. Self-excited square waves with a frequency lower than or close to the resonant frequency of the PT will excite resonant modes inside the transformer. This is achieved by the fundamental frequency of the square waves. Since the PT is operating as a high quality factor (Q) band pass filter (BPF), it filters higher order harmonics out and transfers the fundamental component to its output. The electrical quality factor of the PT is derived as:

$$
Q=\frac{1}{\omega C_{d 2} R_{L}}
$$

where $\omega=1 / \sqrt{L C}$ is the series-resonance angular frequency of the PT [8]. Therefore, the resonant current is considered as a sinusoidal waveform and is described as

$$
i_{\text {res }}(t)=I_{p k}(t) \sin \left(\omega t-\phi_{I}\right)
$$

where $\phi_{I} \in[0, \pi]$ is the current phase angle.

The amplitude of the fundamental resonant current grows with time until it reaches to a certain level that the self-oscillating loop becomes locked at the resonant frequency. Since the amplitude of the sinusoidal waveform at the output of low pass filter (LPF) shown in Fig. 2, becomes greater than the amplitude of the self-induced oscillation waveform, the oscillator operates as a comparator. This allows the comparator's behavior to change from that of an oscillator to that of a true comparator. Therefore, it compares the resonant current with a DC level in order to mark the zero crossing of the current. The loop is designed for the case in which the PT is connected to the resistive matched load [7].

$$
R_{\text {marched }}=\frac{1}{\omega C_{d 2}}
$$

The reasoning behind this design choice is that a matched load is considered to be the worst case scenario for a PT, in terms of achieving soft switching [9]. Zerovoltage switching (ZVS) is a form of soft switching considered in this work. At the matched load, the energy 


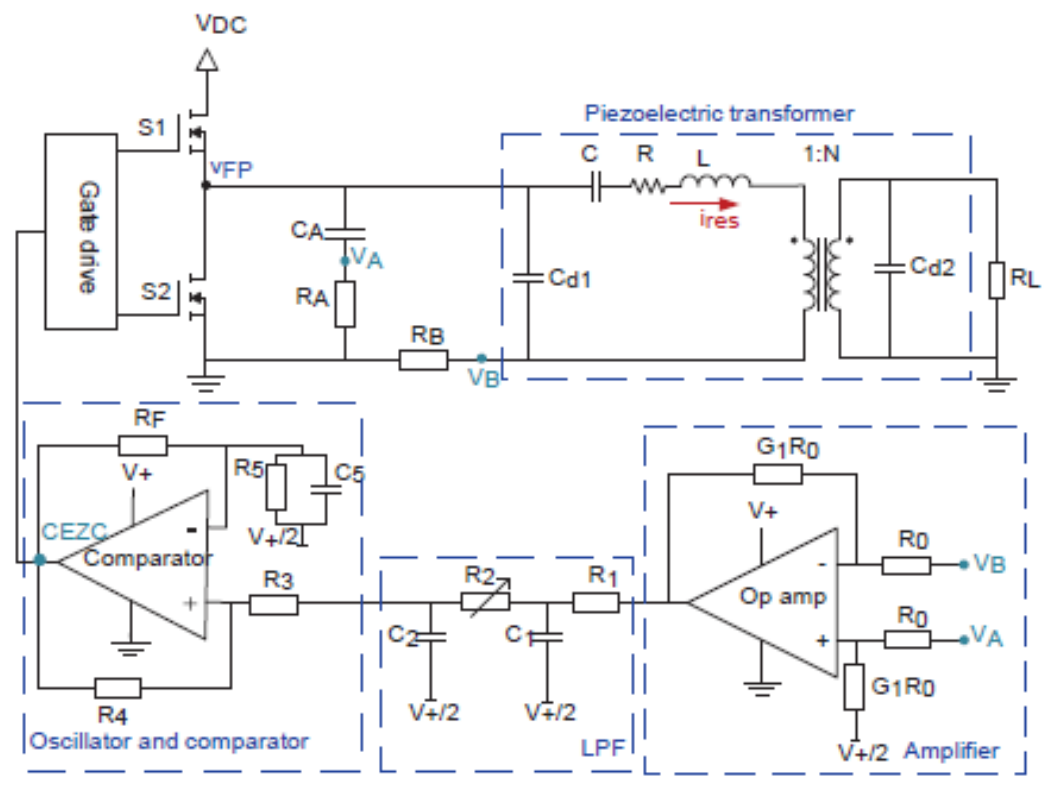

Fig. 1: Circuit design of self-oscillating loop in the PT-based SMPS.

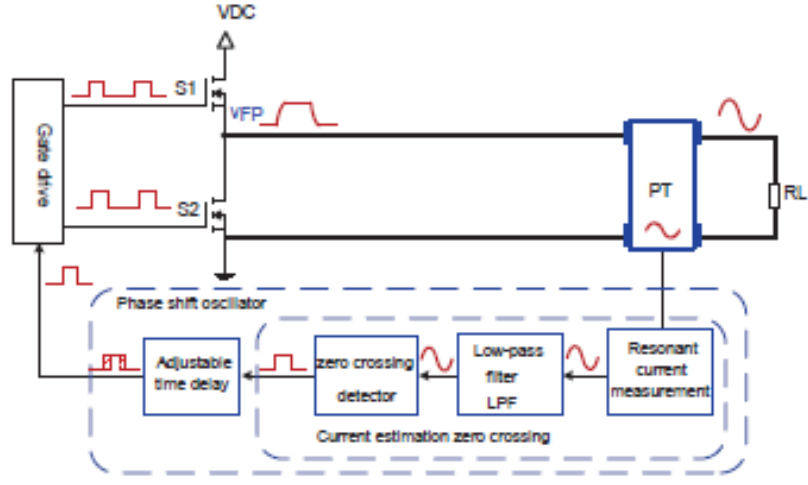

Fig. 2: Block diagram of the self-oscillating loop from the previous research.

transfer through the PT is maximum and therefore its efficiency is maximized as well. This results in a point of minima on the ZVS factor axis [4], [10]. The role of the ZVS factor is to provide the worst case scenario for analyzing PTs in terms of soft switching capability, which means that if the ZVS is achieved for the matched load, it will be obtained for other loads as well [9]. The worst case explanation of the approximated ZVS factor is expressed based on empiric analysis of the ZVS in the following equation [11]:

$$
V_{p}^{\prime}=\left(0.304 \frac{1}{n^{2}} \frac{C_{d 2}}{C_{d 1}}+0.538\right) \cdot(0.585 \eta+0.414)
$$

where $\eta$ is the efficiency of the transformer. $V_{p}^{\prime}$ is the ZVS factor and should be above 1 in order to obtain soft switching. Therefore, the ZVS region is a narrow frequency range above the resonant frequency, where the PT behaves as an inductor and $V_{p}^{\prime}>1$. Furthermore, the ZVS bandwidth of the PT is a ratio of $L / C$ for a constant resonant frequency [9]. Equation (4) is based on a primary analysis of ZVS in order to justify the necessity to design a driver and the self-oscillating loop.

\section{B. Current estimation zero crossing (CEZC)}

The resonant current in the PT is reconstructed within two time intervals. Voltage $V_{B}$ across $R_{B}$ shown in Fig. 1 measures this resonant current while the switches are on. Voltage $\mathrm{V}_{\mathrm{A}}$ across $\mathrm{R}_{\mathrm{A}}$ measures the resonant current during dead time (DT), while both switches are off. The resonant current in this period is supplied by the PT's input capacitor $C_{d 1}$. Therefore, the current passing through $C_{d 1}$ follows the resonant current and can be measured by differentiating the voltage across $C_{d 1}$. This is performed by using $\mathrm{C}_{\mathrm{A}}$ and $\mathrm{R}_{\mathrm{A}}$ as a differentiator. By proper subtraction of these two voltage waveforms through the op-amp, an approximate sinusoidal waveform representing the resonant current will be reconstructed in the output [7], [12], [13].

The estimated current has a $180^{\circ}$ phase shift compared to the resonance current which results in the same zero crossing points. Thereafter, the estimated resonance current is transmitted to a second order LPF which 


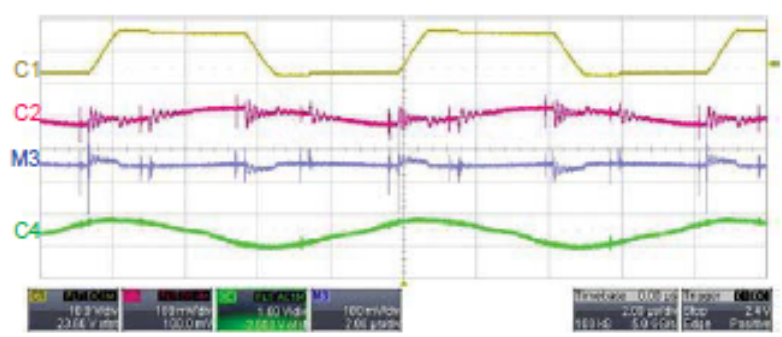

Fig. 3: Reconstruction of the resonant current. $\mathrm{Cl}$ : switching voltage $v_{F} p, \mathrm{C} 2$ : the voltage $\mathrm{V}_{\mathrm{B}}, \mathrm{M} 3$ : the voltage $\mathrm{V}_{\mathrm{B}}, \mathrm{C} 4$ : voltage output of the op-amp Gl. $\left(\mathrm{V}_{\mathrm{A}}-\mathrm{V}_{\mathrm{B}}\right)$

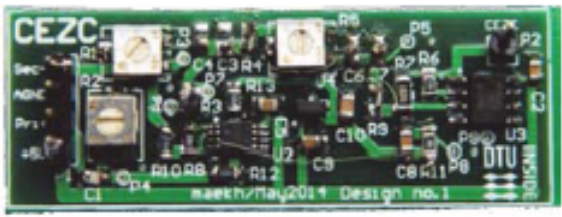

Fig. 4: The CEZC board.

provides an additional phase shift through the feedback loop. The filtered signal is transmitted to the comparator and generates a square wave indicating the zero crossing of the input signal. The square wave signal is fed into the adjustable time delay in order to compensate for the rest of the phase shift to have a total phase shift of $360^{\circ}$ in the entire loop from the input of the gate driver to the output of the feedback loop. In case the switching frequency needs to be decreased, the total phase shift should be increased. Additional phase shift in the loop is added through a digitally-controlled time delay. Since time delay is a positive value, the controlled delay adds an adequate phase shift in order to synchronize the frequency in the next cycle.

\section{Experimental results}

A radial mode PT with Mason's equivalent circuit, shown in Fig. 1, is used, driven by square wave signals with a switching frequency of $118.3 \mathrm{kHz}$, while driving a resistive load of $300 \Omega$. Moreover, reconstructed resonant current from voltages $V_{A}$ and $V_{B}$ is shown in Fig. 3 . The designed board is shown in Fig. 4. Fig. 5 shows experimental waveforms for the self-oscillating loop. Phase shifts and corresponding time delays between stages are measured and shown in Table I. Furthermore, the equivalent parameter values of the PT are measured and shown in Table II.
TABLE I: Phase shifts during one switching cycle in the selfoscillating loop; switching frequency is $118.3 \mathrm{kHz}$ with the time period of $8.45 \mu$ s. HS: high-side control signal, HSGD: high-side gate voltage in the output of the gate driver, $\mathrm{I}_{\text {est: }}$ estimated current in the input of the LPF.

\begin{tabular}{|c|c|c|c|}
\hline Delay & Time [us] & Phase [ $\left.{ }^{0}\right]$ & Duty cycle $\%$ \\
\hline HS $\rightarrow$ HSGD & 1.59 & 67.8 & 18.85 \\
\hline HSGD $\rightarrow \mathrm{L}_{\text {est }}$ & 0.27 & 11.5 & 3.19 \\
\hline $\mathrm{Lex}_{\mathrm{s}} \rightarrow \mathrm{LPF}$ & 6.09 & 259.4 & 72.04 \\
\hline $\mathrm{LPF} \rightarrow \mathrm{CEZC}$ & 0.5 & 21.3 & 5.92 \\
\hline Total & 8.45 & 360 & 100 \\
\hline
\end{tabular}

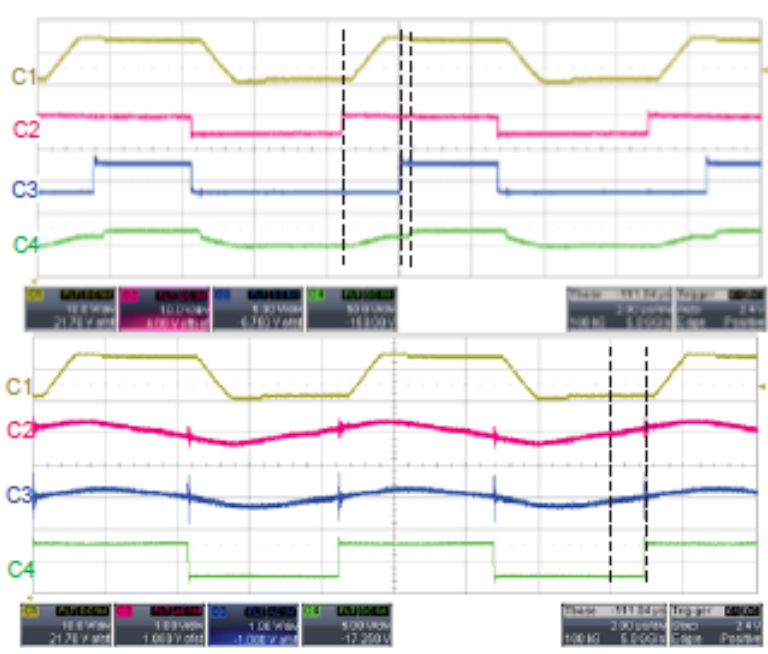

Fig. 5: The self-oscillating loop in the previous research; wave forms from top to bottom show phase shift during one switching cycle. On top: $\mathrm{Cl}$ : the switching voltage $\left(v_{F} p\right)$; $\mathrm{C} 2$ : $\mathrm{CEZC}$; $\mathrm{C} 3$ : input signal to the high-side gate drive; $\mathrm{C} 4$ : high-side gate drive output to the ground. On bottom: $\mathrm{Cl}$ : switching voltage $\left(v_{F P}\right)$; $\mathrm{C} 2$ : estimated resonant current in the output of op-amp; C3: estimated resonant current in the output of the LPF; C4: the CEZC.

TABLE II: PT equivalent parameters

\begin{tabular}{cccc}
\hline \hline Parameter & Value & Parameter & Value \\
\hline$C_{d 1}$ & $3.8 \mathrm{nF}$ & $C_{d 2}$ & $626 \mathrm{pF}$ \\
$C$ & $565 \mathrm{nF}$ & $R$ & $5.6 \Omega$ \\
$L$ & $3.5 \mathrm{mH}$ & $N$ & 3.5 \\
\hline \hline
\end{tabular}

\section{Digitized DELAY LiNE (DDL)}

\section{A. Design considerations}

Initial investigation of the time step resolution shows need for a finely adjust of the total delay in the loop. The result of this investigation showed that there was a change in the amplitude of the PT's output voltage for every $10 \mathrm{~Hz}$ change in the switching frequency. For example, if the operating frequency of $100 \mathrm{kHz}$ increases by $10 \mathrm{~Hz}$, the output voltage shows a consid- 


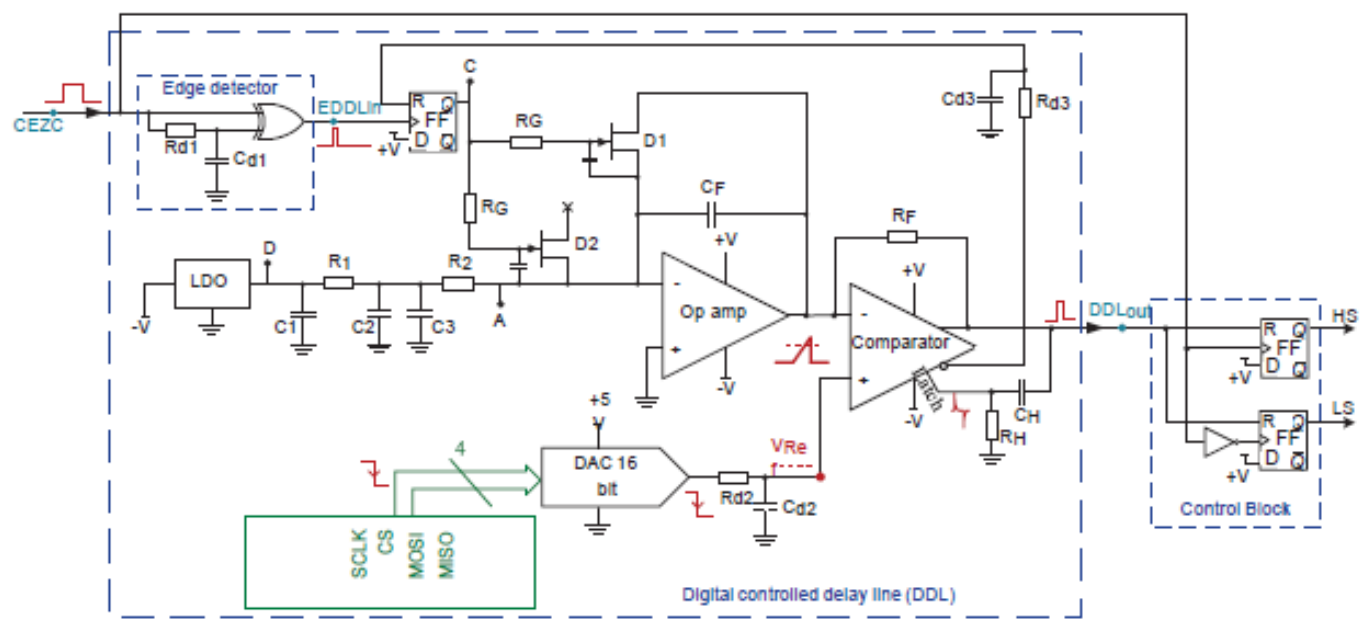

Fig. 6: The electrical circuit of the DDL block, with resolution of $1 \mathrm{~ns}$.

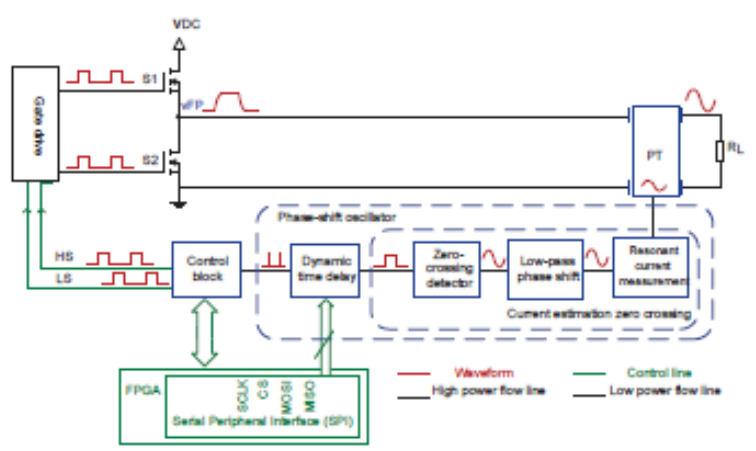

Fig. 7: Block digram of self-oscillating loop with dynamic time delay.

erable change in the amplitude. Therefore, a precision of minimum $10 \mathrm{~Hz}$ in the frequency is required for a dynamic time delay which is equivalent to 1 ns resolution. Fig. 6 shows a simplified version of the circuit used as a control block. Fig. 7 shows the block diagram of the self-oscillating closed-loop together with the contribution of the digital-to-analog converter (DAC) and fieldprogrammable gate array (FPGA). These are used to implement a high-resolution time delay inside the DDL block.

Fig. 8 shows a detailed drawing of the main signal waveforms in the circuit. $v_{F P}(t)$ is the transformer's primary-side voltage while exhibiting soft switching. $i_{\text {res }}(t)$ shows the resonant current of the PT. This current is dependent on the characteristic parameters of the PT and it changes its polarity when either the switches are on or their body diodes conduct. Therefore, depending

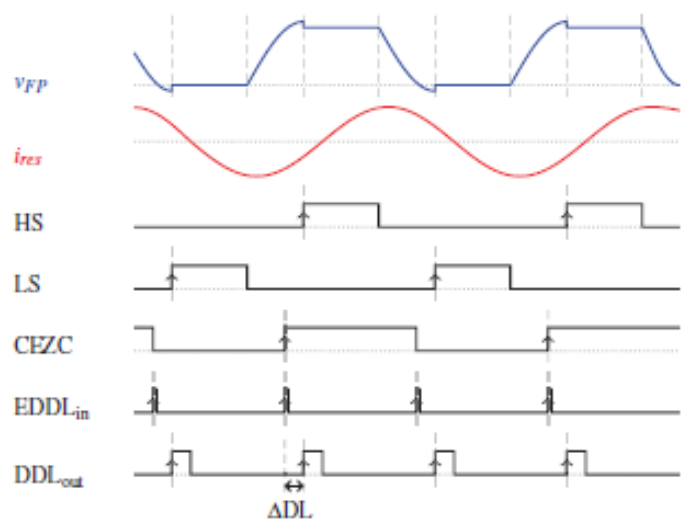

Fig. 8: Signal waveforms with negligible propagation delays in digital gates and the gate driver. $\Delta \mathrm{DL}$ : applied delay with the resolution of Ins

on the operating frequency and temperature of the PT, there is a phase shift between the resonance frequency and the switching voltage, that is defined as $\phi_{I}$ in (2). Consequently, it is important that the circuit is capable of compensating for this phase shift. The estimated resonant current shown in Fig. 7 passes through a $180^{\circ}$ phase shifter, then a LPF and a comparator. This is illustrated in Fig. 8. The output of the CEZC block which has the same frequency as the resonance is then tied to the DDL block. In DDL block, the input signal is first transformed into edge-detecting one-shot pulses $\left(\mathrm{EDDL}_{\text {in }}\right.$ ) which are then used as a clock source for the flip-flop (FF). The 


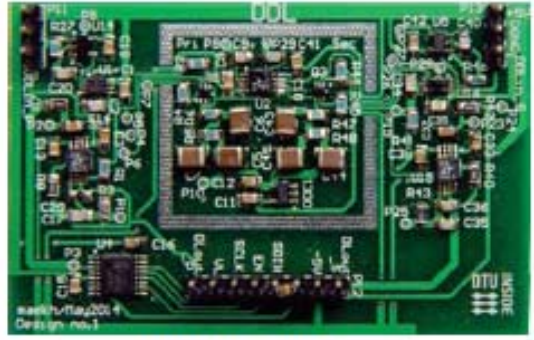

Fig. 9: The DDL board

signal from this $\mathrm{FF}$ is then used to reset the hardware integrator present in the circuit. Namely, when the input signal edge-triggers the $\mathrm{FF}$, the feedback capacitor in the op-amp's feedback starts charging, thereby creating a fixed voltage slope. This is then compared to the variable reference voltage provided by the DAC. Thereafter, the complementary output of the comparator resets the FF which consequently turns the MOSFET, D1, on and discharges the feedback capacitor, thereby resetting the integrator. Since the input pulse triggers the start of the integration, the variable reference provided to the comparator by the DAC coupled with the voltage slope work together to create a time-delayed version of the input pulse which is proportional to the DAC output value. The output of the comparator is then latched for a short time by its own output through a high-passed signal to its latch pin, resulting in one-shot pulses at the output of the DDL.

The output of the DDL is capable of prolonging the switching period by changing the on time of the switches $\left(\mathrm{T}_{\mathrm{on}}\right)$. The high-side and low-side switches are turned on by rising edges of CEZC and $\overline{\mathrm{CEZC}}$ signal, respectively, which are used as clock inputs to the control block FFs. The output of the DDL block is then used to reset the FFs, thereby turning the switches off. Dead time (DT) is fixed for a specific design regarding a certain PT and switching frequency. Since this DT is fixed, by adjusting the time-delay for turning the switches off, the frequency of self-oscillation changes. DDL block delays its input (CEZC) to compensate the rest of phase shift through loop to reach $360^{\circ}$. The propagation delay in the control block is assumed negligible in the waveforms shown in Fig. 8 .

\section{B. Experimental results}

A prototype has been built and tested. Fig. 9 shows designed modular board for DDL block. A radial mode PT with Mason's equivalent circuit is used, driven by

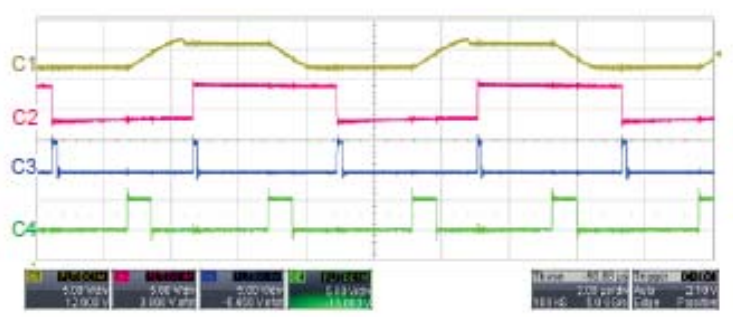

Fig. 10: Measurement: Signal waveforms where dynamic delay is applied; $\mathrm{Cl}$ : switching waveform $\left(v_{F P}\right) ; \mathrm{C} 2$ : $\mathrm{CEZC}$ as input of the DDL block; $\mathrm{C} 3$ : one-shot pulse EDDL in $\mathrm{C} 4$ : output of the DDL. block (DDL -aut ).

TABLE III: One cycle phase shift measured in the self-oscillating loop, HS: high-side control signal, HSGD: high-side gate voltage in the output of the gate driver, lest: estimated current in the input of the LPF.

\begin{tabular}{|c|c|c|c|}
\hline Delay & Time [us] & Phase [0] & Duty cycle \% \\
\hline HS $\rightarrow$ HSGD & 0.28 & 11.94 & 3.3 \\
\hline $\mathrm{HSGD} \rightarrow \mathrm{I}_{\text {est }}$ & 6.6 & 281.52 & 78.2 \\
\hline $\mathrm{b}_{\mathrm{st}} \rightarrow \mathrm{LPF}$ & 1.56 & 66.5 & 18.5 \\
\hline Total & 8.44 & 360 & 100 \\
\hline D:EDDL in $_{1} \rightarrow$ DDL $_{o d}$ & 2.5 & 106.6 & 29.6 \\
\hline
\end{tabular}

square wave signals with a switching frequency of 118.3 $\mathrm{kHz}$ and a resistive load of $300 \Omega$. Furthermore, the equivalent parameter values of the PT are measured and shown in Table II. The input signal waveform of DDL circuit i.e. CEZC and one-shot input pulse together with the output of DDL are shown in Fig. 10. Table III shows measured phase shifts and corresponding time delays between stages.

\section{Conclusion}

General operation of a self-oscillating loop for piezoelectric transformer-based power converters is explained. The designed circuit for operating the transformer together with experimental results are provided. The circuit is based on a new idea for compensating and controlling the phase shift for the self-oscillating in order to achieve and maintain soft switching. This is combined with a resonant current estimation that is able to start and maintain the circuit oscillation. The operation of and cooperation between the analog resonance current estimator and digital phase shift adjustment are presented in detail, together with some insight into the performance of the designed circuit. The concept is proven through the experimental results. 1 ns time step resolution is sufficient for adjusting the phase shift of the loop. The designed circuit is able to follow changes in the resonant frequency of the PT in every cycle. 


\section{ACKNOWLEDGMENT}

The authors would like to thank Noliac A/S for supplying the prototype of a piezoelectric transformer as well as "The Danish National Advanced Technology Foundation" for financial support.

\section{REFERENCES}

[1] M. Ekhtiari, Z. Zhang, and M. A. E. Andersen, "State-of-the-art piezœelectric transformer-based switch mode power supplies," 40th Annual Conference of the IEEE Industrial Electronics Sociery, IECON2014, pp. 5072-5078, 2014.

[2] E. Horsley, M. Foster, and D. Stone, "State-of-the-art piezoelectric transformer technology," in Power Electronics and Applications, 2007 European Conference on, Sept 2007, pp. $1-10$.

[3] A. V. Carazo, "50 years of piezoelectric transformers. trends in the technology," in Materials and Devices for Smant Systems Symposium, ser. MRS Proceedings, vol. 785, 2003.

[4] C. y. Lin, "Design and analysis of piezoelectric transformer converters," Ph. D. Dissertation, Virginia Tech, 1997.

[5] M. A. E. Andersen, K. Meyer, M. S. Rodgaard, and T. Andersen, "Piezoelectric power converter with bi-directional power transfer," US patent 61/567,924 and 2013, WIPO, WO2013083679-A1, 2011.

[6] M. Rodgaard, "Bi-directional piezoelectric transformer based converter for high-voltage capacitive applications," in Applied Power Electronics Conference and Exposition (APEC), 2015 IEEE, March 2015, pp. 1993-1998.

[7] M. Rodgaard, M. A. E. Andersen, T. Andersen, and K. Meyer, "Self-oscillating loop based piezoelectric power converter," US patent 61/638,883 and WIPO, WO2013083678-A2, 2011.

[8] G. Ivensky, I. Zafrany, and S. Ben-Yaakov, "Generic operational characteristics of piezoelectric transformers," Power Electronics, IEEE Transactions on, vol. 17, no. 6, pp. 1049-1057, Nov 2002.

[9] K. Meyer, M. Andersen, and F. Jensen, "Parameterized analysis of zero voltage switching in resonant converters for optimal electrode layout of piezoelectric transformers," pp. 2543-2548, June 2008.

[10] R. L. Lin, "Piezoelectric transformer characterization and application of electronic ballast," Ph.D. Disserration, Virginia Polytechnic Institute and State University, 2001.

[11] M. Rodgaard, T. Andersen, and M. Andersen, "Empiric analysis of zero voltage switching in piezoelectric transformer based resonant converters," pp. 1-6, March 2012.

[12] T. Andersen, "Piezoelectric transformer based power supply for dielectric electro active polymers," Ph.D. Thesis, Deparment of Electrical Engineering, Technical University of Denmark, 2012.

[13] M. S. Rodgaard, "Piezoelectric transformer based power converters; design and control," Ph.D. Thesis, Deparment of Electrical Engineering, Technical University of Denmark, 2012. 